Revue européenne des sciences sociales

European Journal of Social Sciences

XLIV-134 | 2006

Quel(s) défi(s) pour les sciences sociales à l'heure de la mondialisation?

\title{
Les illusions de la techno-démocratie de marché mondialisée
}

Serge Latouche

\section{CpenEdition}

Journals

Édition électronique

URL : http://journals.openedition.org/ress/297

DOI : $10.4000 /$ ress.297

ISSN : 1663-4446

Éditeur

Librairie Droz

Édition imprimée

Date de publication : 1 juin 2006

Pagination : 181-192

ISBN : 9-782-600-01095-5

ISSN : 0048-8046

Référence électronique

Serge Latouche, "Les illusions de la techno-démocratie de marché mondialisée », Revue européenne des sciences sociales [En ligne], XLIV-134 | 2006, mis en ligne le 14 octobre 2009, consulté le 21 avril 2019. URL : http://journals.openedition.org/ress/297 ; DOI : 10.4000/ress.297

(c) Librairie Droz 


\title{
LES ILLUSIONS DE LA TECHNO-DÉMOCRATIE DE MARCHÉ MONDIALISÉE ${ }^{1}$
}

\begin{abstract}
Ce qui est ici en question est la critique du projet technicien qui caractérise la société industrielle. J'entends par là la volonté de remplacer le tissu social, les liens de solidarité qui constituent la trame d'une société, par une fabrication; le projet inédit de produire les relations des hommes à leurs voisins et à leur monde comme on produit des automobiles ou des fibres de verre. L'autoroute, le rein artificiel et l'Internet ne sont pas seulement des objets ou des systèmes techniques; ils trahissent un certain type de rapport instrumental à l'espace, à la mort et au sens. C'est ce rapport instrumental, le rêve de maîtrise qu'il recouvre que la critique se doit d'analyser pour en mesurer les effets délétères.
\end{abstract}

Jean-Pierre Dupuy ${ }^{2}$

En 1994, lorsque j'écrivais La mégamachine, les biotechnologies commençaient tout juste à se répandre et les semences transgéniques n'étaient pas encore l'objet d'un débat passionné, le clonage restait du domaine de la science fiction, les nanotechnologies étaient toujours dans les limbes et les rues françaises étaient libres de téléphones portables et de leur incivilité congénitale... Pourtant, 15 ans après, sauf quelques détails, je n'ai rien trouvé à y changer. Il y a là un paradoxe. L'extraordinaire dialogue entre Theuth, le dieu Ibis inventeur du nombre, du calcul, de la géométrie et de l'astronomie, «sans parler du trictrac et des dès », mais surtout de l'écriture, et le roi Thamous, tel qu'il est rapporté dans le Phèdre de Platon, nous permet de le comprendre.

«Voilà, dit Theuth en présentant l'invention de l'écriture, Ô roi, qui procurera aux Egyptiens plus de science et plus de souvenirs; car le défaut de mémoire et le manque de science ont trouvé leur remède!» A quoi le roi répondit: «O Theuth, découvreur d'arts sans rival, autre est celui qui est capable de mettre au jour les procédés d'un art, autre celui qui l'est, d'apprécier quel en est le lot de dommage ou d'utilité pour les hommes appelés à s'en servir! Et voilà maintenant que toi, en ta qualité de père des lettres de l'écriture, tu te plais à doter ton enfant d'un pouvoir contraire de celui qu'il possède. Car cette invention, en dispensant les hommes d'exercer leur mémoire, produira l'oubli dans l'âme de ceux qui en auront acquis la connaissance; en tant que, confiants dans l'écriture, ils chercheront

La première partie de cette contribution reprend en partie l'introduction de la nouvelle édition de notre livre La mégamachine, Paris, La Découverte, 2004.

2 Jean-Pierre Dupuy, Pour un catastrophisme éclairé, Paris, Seuil, 2003, p. 28. 
au dehors, grâce à des caractères étrangers, non point au dedans et grâce à euxmêmes, le moyen de se ressouvenir; en conséquence, ce n'est pas pour la mémoire, c'est plutôt pour la procédure du ressouvenir que tu as trouvé un remède. Quant à la science, c'en est l'illusion, non la réalité, que tu procures à tes élèves: lorsqu'en effet, avec toi, ils auront réussi, sans enseignement, à se pourvoir d'une information abondante, ils se croiront compétents en une quantité de choses, alors qu'ils sont, dans la plupart, incompétents; insupportables en outre dans leur commerce, parce que, au lieu d'être savants, c'est savants d'illusion qu'ils seront devenus !» ${ }^{3}$. Les réflexions du roi ressemblent étrangement à celles faites de nos jours par le théologien Ivan Illich quand celui-ci, par exemple, dénonce «La condition humaine» actuelle, dans laquelle toutes les technologies deviennent si envahissantes qu'on ne saurait plus trouver de joie que dans ce que j'appellerais un «techno-jeûne » ${ }^{4}$. S'appuyant sur Jacques Ellul, il développe le thème de l'humiliation de l'homme par la technique. "Nos pieds, dit Illich, qui nous avaient été donnés pour accomplir notre pèlerinage sur terre sont atrophiés au point de ne plus servir que d'outils pour pousser les freins ou l'accélérateur ». La technique nous transforme ainsi en outil de son propre développement. C'est la mise en «dis-valeur» de l'homme, corollaire de la mise en valeur/destruction de la nature.

Même si la société grecque de Platon ignorait tout autant la société technicienne que celle d'Aristote la société de marché, qu'elle n'était ni capitaliste, ni thermo-industrielle, les deux grands philosophes avaient mis le doigt sur quelque chose d'essentiel dans les dimensions marchandes et techniciennes de ce que serait la modernité. Si le texte de Platon (pas plus que celui d'Aristote sur la chrématistique) n'a pas vieilli c'est qu'il ne s'agit pas de textes techniques de technique ou d'économie, mais d'une réflexion philosophique sur la technique et l'économie. Si le monde de Platon et d'Aristote est à des années-lumière de celui de G. W. Bush, les questions que soulèvent ces penseurs restent les nôtres pour l'essentiel, et au niveau philosophique, ils sont encore nos contemporains. En ce qui concerne l'univers des techniques, les mythes, relayés par les utopies, puis la science-fiction ont déjà exploré depuis fort longtemps de multiples aspects de la mégamachine en voie de réalisation et que j'ai appelée «Occident». L'Occident, en effet, aujourd'hui nous apparaît comme une machine vivante, mi-mécanisme mi-organisme, dont les rouages sont des hommes et qui, pourtant, autonome par rapport à eux dont elle tire force et vie, se meut dans le temps et l'espace suivant son humeur propre. Ce qui n'existait pas encore il y a dix ou quinze ans, était déjà prévisible et contenu en puissance. Les mises en garde sur les bricolages du vivant et la fabrication de cybernanthropes ou sur la perte des libertés par les contrôles techniques restent d'actualité plus que jamais puisque les dangers n'ont fait que se renforcer. Si les exemples se renouvèlent, les logiques demeurent inchangées. Il en est ainsi parce que nous nous situons sur un plan où le progrès n'existe pas 5 .

\footnotetext{
Platon, Phèdre (pour la présente traduction, Euvres complètes, Paris, Gallimard, Bibliothèque de La Pléiade, 1950, t. 2, p. 75). Voir aussi Neil Postman, Technopoly. The Surrender of Culture to Technology, New York, A. Knopf, 1992, et pour la présente citation, Torino, Bollati Boringhieri, 1993, p. 12.

«L'origine chrétienne des services », in La perte des sens, Paris, Fayard, 2004, p. 43.

5 Maurice Merleau-Ponty nous le rappelle avec raison, voir L'Eil et l'Esprit, Paris, Galllimard, 1964, p. 91-93.
} 
Ainsi, les analyses de La mégamachine de 1994 et de L'occidentalisation du monde de 1989 semblent pour l'essentiel pouvoir être reprises sans changement en 2005. La récente publication dans la collection du MAUSS du savant ouvrage d'Andrew Feenberg, (Re)penser la technique, nous donne l'occasion de faire le point sur la possibilité de «rénover » la modernité par l'apport des nouvelles technologies, en particulier sur le projet de la démocratie, et de tester ainsi la validité des analyses de Jacques Ellul et d'Ivan Illich ${ }^{6}$.

\section{PEUT-ON RÉNOVER LA MODERNITÉ TECHNICIENNE?}

La mégamachine, autrement dit l'Occident, déjà défini dans L'occidentalisation du monde, comme mégamachine anonyme, n'est autre que la grande société, le monde des gagnants de «La planète». Si le technique y occupe une place centrale, cela tient surtout au fait qu'il est la forme en laquelle s'incarne le mieux l'imaginaire du progrès et que celui-ci joue un rôle structurant dans la modernité. Ellul dénonçait un totalitarisme technicien conduisant à une société totalitaire. La société technicienne est poussée certainement à s'enfermer totalement sur ellemême; tout problème étant technique, ne peut trouver de solution que technique. Ainsi, la pollution engendrée par la technique réclame plus de technique pour résoudre les problèmes qu'elle pose. On songe, par exemple, à créer des bactéries nouvelles ou autres xénoorganismes pour dévorer ou recycler les déchets et les scories du technocosme. La technique engendre des situations telles qu'il paraît bien impossible de s'en sortir sans recourir à encore plus de technique. Internet participe peut-être des solutions techniques à la destruction du lien social due à la société technicienne et contribue à remédier à la «solitude de l'homme moderne» en permettant à des déracinés d'entrer dans des communautés virtuelles et de s'éclater dans le deuxième monde du cyberespace...

Toutefois, faire pour cela d'Ellul un technophobe et un représentant de «l'essentialisme de la technique» montre de la part de Feenberg (mais aussi de beaucoup de critiques), une certaine incompréhension. En particulier, ce reproche témoigne de la méconnaissance de la distinction, capitale chez lui, entre «la technique », c'est-à-dire le système technicien, qui correspond à la modernité, avec «les techniques». Comme le souligne justement Ivan Illich, qui se considère comme un disciple du maître, le premier apport de Jacques Ellul «c'est l'impossibilité de comparer la technique moderne et ses terrifiantes conséquences avec la culture matérielle d'une autre société quelle qu'elle soit» ${ }^{7}$. Affirmer qu'il y a «une essence de la technique, et une seule, et qu'elle est responsable des problèmes principaux de la civilisation moderne » vaut peut-être pour Heidegger, mais pas pour l'auteur du système technicien, même si le rôle totalitaire attribué à celui-ci reste discutable. L'idée maîtresse de Feenberg est que «le développement technique est un processus social et il ne peut se comprendre qu'en tant que tel ${ }^{8}$.

\footnotetext{
- Andrew Feenberg, (Re)penser la technique. Vers une technologie démocratique, p. 217.

Hommage d'Ivan Illich à Jacques Ellul, in Perte des sens, Paris, Fayard 2004, p. 154.

s op. cit., p. 52.
} 
«J'affirme (...) que les technologies ne sont pas des dispositifs physiques que l'on peut extraire de valeurs sociales contingentes. La technique incorpore toujours le social dans sa structure ${ }^{9}$. Certes, mais Ellul ne dit pas autre chose et nous le suivons aussi totalement sur ce point. Paradoxalement, Feenberg lui-même n'échappe pas à un certain «substantialisme» de la technique qui constitue pourtant sa bête noire. Ainsi la phrase finale du livre, «Dans cet avenir, la technique ne sera pas un destin qu'il faut accepter ou rejeter, mais un défi à la créativité politique et sociale », attribue à une technique «essentielle» un rôle clef même si nous entendons bien que pour lui, elle «soit replacée dans le cadre du mouvement démocratique de l'histoire ». Ellul, certes, dans certaines formulations, n'y échappe pas non plus (et nous-même aussi très probablement), tant il est vrai qu'il est difficile d'échapper à certaines manières de parler héritées. Plus, «notre » Mauss qui, presque seul entre tous, y a échappé en ce qui concerne l'économie, assumant un nominalisme/culturalisme sans faille, succombe au mirage du «primat du technique $(. .$.$) indépendant et cause». C'est qu'il voit dans les techniques «un$ compromis entre la nature et l'humanité $»^{10}$. Autrement dit, un invariant universel et transhistorique. Et pourtant, nous ne pouvons que souscrire pour l'essentiel à l'analyse critique du substantialisme à laquelle procède Feenberg :

Le substantialisme identifie la technique en général avec la technique occidentale moderne. Dans les techniques modernes, il y a assurément des réussites universelles dont nombre d'entre elles furent à l'origine empruntées à d'autres civilisations. Cependant, la forme particulière sous laquelle ces réussites ont été obtenues en Occident incorpore des valeurs qui, loin d'être universelles, appartiennent à une culture et à un système économique bien définis. Ainsi l'erreur du substantialisme ne réside-t-elle pas tant dans sa description de la technique moderne que dans son incapacité à en reconnaître la contingence historique ${ }^{11}$.

La principale différence entre l'approche de Feenberg et la nôtre (et aussi celle d'Ellul) c'est qu'il veut intégrer la critique des thèmes techniques à la philosophie «sans perdre l'espace conceptuel qui permet d'imaginer une reconstruction radicale de la modernité ${ }^{12}$. Autrement dit, sauver la modernité ou mettre en place des «modernités alternatives ». Pour ce faire, pense-t-il, il suffirait de réglementer «le cadre culturel de l'économie» pour rendre la modernité tout à fait présentable, alors que nous pensons qu'il faut en sortir et sortir en même temps de l'économie qui lui est consubstantielle. Sans doute, n'a-t-il pas tort d'insister sur «l'énorme flexibilité des systèmes techniques» ou sur cette «malléabilité de la technique», sous-estimée par Ellul. Il y a incontestablement des techniques qui rendent des services aux opprimés dans leur combat contre leurs oppresseurs. Il y a des techniques plus conviviales que d'autres, même dans notre système technicien. La machine à coudre individuelle inventée par Singer par amour pour son épouse,

${ }^{9}$ Op. cit., p. 202.

${ }^{10}$ Voir l'éclairant article de François Vatin, «Mauss et la technologie», Revue du M.A.U.S.S., $n^{\circ} 23,2004$, p. 423-424. Dans son commentaire, François Vatin en rajoute une louche: «La technique lui apparaît en effet comme le moteur du processus de la 'Civilisation' qu'il conçoit au singulier dans la destinée prométhéenne de l'humanité» (Ibid., p. 425).

${ }_{11}$ Op. cit., p. 217.
${ }^{12}$ Op. cit., p. 39. 
comme le rappelle Ivan Illich, l'est infiniment plus qu'une centrale atomique congénitalement porteuse d'hétéronomie ${ }^{13}$. La bicyclette est de même devenue une merveille technique favorisant l'autonomie et préservant l'environnement, sans commune mesure avec les $4 / 4 \ldots$ Toutefois, la question n'est pas là. Elle est qu'on ne peut pas juger une technique isolément de son contexte, c'est-à-dire de la société qui l'a produite. De la technique moderne on doit dire qu'il ne s'agit pas d'un outil neutre, encore moins d'un outil émancipateur en soi ou qui participerait d'un projet global d'émancipation de l'homme; on ne peut pas dire non plus qu'elle émane d'une culture à objectif extra technoéconomique, comme les outils produits dans les sociétés précapitalistes, préindustrielles, prémodernes... L'existence d'un outil convivial et autonome comme ceux précédemment cités est une heureuse exception dans l'univers de la technoscience. De là à penser que grâce aux «polémiques techniques» et aux «dialogues innovants» qui sont devenus «des dimensions incontournables de la vie politique contemporaine», on puisse déboucher sur des «appropriations créatives» significatives, il y a de la marge. Peut-être faut-il voir là un exemple de cette fraîcheur un peu naïve qui sépare la jeune Amérique de la vieille Europe. A moins que «La peur pathétique d'apparaître comme dépassé par quoi que ce soit, peur qui tient lieu de pensée chez la plupart des intellectuels de gauche (finisse) par aboutir aux noces actuelles de l'avenir radieux et du cybermonde... ${ }^{14}$

Nous, Européens sceptiques, croyons savoir que derrière la mise en scène de la démocratie procédurale, ce sont les lobbies qui font les lois et la loi... La «flexibilité interprétative de la technique» et «l'invention participative» nous semblent, à nous aussi néanmoins, très importantes pour limiter les dégâts du présent et préparer un autre futur, mais cet heureux avènement ne nous paraît possible qu'en changeant de cadre. Tout est dans ce changement. Il ne s'agit donc pas d'une «opposition radicale à la technique», mais bien d'une opposition radicale à la société de marché. «Il est possible que nous puissions encore établir une société démocratique où le progrès technique servira le progrès de la communication.» ${ }^{15}$ Espérons-le, mais cela a quelques relents suspects de cette «télédémocratie » qui incarne les illusions des «rénovateurs » de la modernité ${ }^{16}$.

\section{LES APORIES DE LA TÉLÉDÉMOCRATIE}

La télédémocratie est un thème à la mode. Il existe aujourd'hui une utopie internet (déjà dépassée par l'utopie nanotechnologie), illustrant l'utopie technicienne qui court depuis l'origine de la modernité, de Francis Bacon à Saint Simon et tous les prophètes de l'industrialisme. Il a existé aussi dans les années '70 une utopie «puce électronique». Il n'est pas inutile d'en rafraîchir la mémoire, car il

${ }^{13}$ Ivan Illich, La convivialité, Paris, Seuil, 1973.

${ }^{14}$ Jean-Claude Michéa, «Révolte et conservatisme: les leçons de 1984 », Revue du M.A.U.S.S., n 9, 1997, p. 202.

15 Feenberg, op. cit., p. 108.

${ }^{16}$ Pierre Lévy, «La cyberculture en question: critique de la critique », Revue du M.A.U.S.S., n 9, 1997. 
s'agit toujours du même plat qu'on nous sert indéfiniment réchauffé. En ce temps là, JJSS (Jean-Jacques Servan Schreiber) avait prédit que le microprocesseur allait sauver le tiers-monde et supprimer le sous-développement. On sait ce qu'il en est advenu...

Toute technique est porteuse de rêve et de phantasme, mais les techniques médiatiques l'ont sans doute été plus que toutes les autres. Déjà, l'invention de l'imprimerie avec Guttenberg a été un formidable vecteur de la réforme. La radio, la télé, la vidéo, le Minitel, avant internet, ont été des lieux d'espoirs et d'expériences sociales intéressantes.

\section{Brecht disait en son temps :}

La radio pourrait être le plus formidable appareil de communication qu'on puisse imaginer pour la vie publique, un énorme système de canalisation, ou plutôt elle pourrait l'être, si elle savait non seulement émettre, mais recevoir, non seulement faire écouter l'auditeur, mais le faire parler, ne pas l'isoler, mais le mettre en relation avec les autres. Il faudrait que la radio, abandonnant son activité de fournisseur, organise cet approvisionnement par les auditeurs eux-mêmes ${ }^{17}$.

Internet réalise précisément cette possibilité.

Le développement des radios locales battant en brèche, le monopole public des ondes en France a été un mouvement porteur d'espoir. La guérilla qui s'est déchaînée entre 1977 et 1981 a abouti à la fin du monopole public, mais la part des mouvements sociaux s'est rétrécie comme une peau de chagrin et l'on a assisté à des O.P.A. à coup de millions sur les fréquences les plus convoitées et, pour finir, à la mainmise du groupe Hersant sur l'essentiel.

S'il n'y a pas eu en France de mouvements citoyens comparables pour la télé, et que la guerre des chaînes est restée une affaire de professionnels et de spécialistes, ici ou là (au Québec en particulier), des expériences et des espérances très intéressantes de télévision citoyenne de quartier se sont produites.

La vidéo, par son coût modeste et ses facilités d'utilisation, a fait renaître cette visée d'une autre télévision, décentralisée, permettant aux récepteurs de devenir émetteurs. On trouve déjà cette composante clef de l'offensive du multimédia, à savoir l'idéologie de l'interactif. "La vidéo comme transgression en faisant éclater formes et contenus de la télévision des familles, écrit Yvonne MignotLefebvre, était porteuse de ces espoirs ${ }^{18}$. Les médias bon marché et populaires servent de véhicule à des mouvements contestataires. «Les modèles de société sont rejetés, note encore Yvonne Mignot-Lefebvre, mais nous pouvons constater que les technologies d'information sont utilisées sans tabou pour renforcer les patrimoines organisationnels naissants: informatique, téléphone mobile, réseau internet, cassettes vidéo...» ${ }^{19}$. C'est le cas en particulier avec les mouvements

${ }_{17}$ Brecht, 1970, p. 137, cité dans « Multimédia et communication à usage humain », Dossier pour un débat, $\mathrm{n}^{\circ}$ 56, Paris, Fondation pour le progrès de l'homme, 1996, p. 145.

18 Yvonne Mignot-Lefebvre, «Place de la communication dans les enjeux de l'autonomie. Audiovisuel, nouvelles technologies de l'information et changement social », I, thèse, Université de Paris X, 1997, p. 129.

${ }^{19}$ Ibid., p. 339. 
islamistes qui diffusent les prêches des imams et autres mollah en cassettes audio ou vidéo. Le rôle de la diffusion clandestine des cassettes de Khomeny dans la révolution iranienne n'a pas été négligeable.

L'important et la nouveauté, c'est en fait la baisse des coûts. la communication planétaire instantanée existe en effet depuis plus d'un siècle. Samuel Morse, en 1838 déjà, avait imaginé de «faire de l'Amérique un village» grâce aux liaisons télégraphiques ${ }^{20}$. Depuis le début du siècle, dans différents pays, des expériences d'écoute en direct de concerts, d'envoi de nouvelles et d'informations diverses grâce au téléphone domestique ont été entreprises. Dès 1909, A.T.T. décrivait son réseau comme «une autoroute de la communication $»^{21}$. Toutefois, le fantastique abaissement des coûts rend accessible, désormais, même aux groupements paysans d'Amazonie l'échange interactif! La planétarisation de l'information, qui a eu un rôle non négligeable dans les événements qui ont entraîné la chute du mur de Berlin, fait rêver (et pas seulement à gauche) d'une démocratie sans frontière. «La libre circulation de l'information génère un besoin de démocratie» (exemple Taiwan ou le Chili). La NII (National Information Infrastructure) pourrait aider à créer un «parlement électronique ${ }^{22}$, déclarait le vice président $\mathrm{Al}$ Gore. L'idée d'une télédémocratie avec ses citizenet est en l'air, elle a été développée en France par Pierre Levy. Il y a des façons très diverses de concevoir cette télédémocratie. Pour certains, c'est tout simplement ce mélange d'agora électronique et de supermarché qui a la faveur des pouvoirs publics américains et qui correspond à une alliance objective des anarchistes et des ultra-libéraux. Bill Gates qui a commencé sa carrière au sein du courant libertaire est tout à fait représentatif d'une génération d'entrepreneurs libéraux néo-anarchistes. Pour d'autres, internet représente la possibilité de retrouver une forme de démocratie directe, pour d'autres encore, c'est la base d'organisation d'une «société civile planétaire » constituant un contre-pouvoir à l'image des puissants réseaux écologiques aux Etats-Unis. Enfin, pour ceux qui sont restés trop longtemps devant leur écran, comme dit Walter Kirn, et ont attrapé «une éruption de millénarisme high-tech» ou ont succombé à «une attaque schizoïde fin de siècle», c'est l'entrée dans une cybersocialité voire un cybercommunisme pour internautes où, grâce à la numérisation de toutes les sensations, on pourrait bientôt vivre la totalité de ses phantasmes $^{23} \ldots$ Internet peut carrément faire délirer. Mark Dery dénonce avec humour la «rhétorique du sublime technologique et le mythe technotranscendental»d'une ultrahumanité. «Ces visions d'une cyberassomption, commente Roland Jaccard, sont une ruse fatale qui nous fait oublier le saccage de la nature, les déchirures du lien social et l'abîme qui se creuse entre l'élite technocratique et les masses souspayées $»^{24}$.

${ }^{20}$ Chiara Ottaviano, Mezzi per comunicare. Storia, società e affari dal telegrafo al modem, Torino, Paravia, 1997, p. 142.

${ }^{21}$ Ibid., p. 118.

22 «Multimédia et communication à usage humain», Dossier pour un débat, $\mathrm{n}^{\circ} 56$, Paris, Fondation pour le progrès de l'homme, 1996, p. 29 et 69.

${ }_{23}$ Jean-Louis Weissberg, «Internet, un récit utopique», Terminal, n 71/72 (Spécial Internet), 1997.

${ }^{24}$ Mark Dery, Vitesse virtuelle, Paris, Ed. Abbeville, 1997, cité par Roland Jaccard, « Mythologies de la cyberculture», Le monde des livres, 6.6.1997. 
Internet est-il le loup dans la bergerie qui permettrait de battre en brèche le quasi monopole médiatique du Nord et singulièrement des transnationales à dominante nord-américaines? «Il ne manquent pas de gens, note Chiara Ottaviano, qui affirment que les pays du tiers monde pourraient y trouver des avantages décisifs, du fait qu'ils auraient facilement à disposition ce qui constitue aujourd'hui la principale matière première: la connaissance et l'information ${ }^{25}$. Il en est ainsi pour Christian Huitema, chercheur en informatique et membre de l'Internet Activities Board, auteur d'un ouvrage intitulé Et Dieu créa l'Internet (Ed. Eyrolles). «L'internet, déclare-t-il dans une interview réalisée par@mail, peut être une chance extraordinaire pour les pays 'en développement'. Le réseau doit permettre à l'étudiant africain d'accéder à la bibliothèque de la Sorbonne, au commerçant indien de proposer ses marchandises sans intermédiaire à des clients européens. Tout cela va dans le sens de plus d'égalité $»^{26}$.

Remettons les pendules à l'heure. En ce qui concerne le net, l'immense majorité des ordinateurs se trouve au Nord, l'immense majorité des sites et des serveurs aux Etats-Unis. Loin de résorber l'écart Nord/Sud, le multimédia risque d'avoir l'effet contraire ${ }^{27}$.

Là encore, on ne peut s'empêcher de ressentir une impression de «déjà vu». On peut rappeler les espoirs et les déboires du «nouvel ordre mondial de l'information et de la communication», préconisé en 1977 par l'Unesco et la commission Mac Bride. On ne peut que souscrire à ce que disait alors un grand intellectuel africain, Babakar Sine:

L'essentiel n'est-il pas de tout faire pour que l'avènement de la civilisation de l'audiovisuel ne se traduise pas par l'évincement systématique et aveugle de cette culture africaine de base, qui se développe de façon vivante dans les communautés (les villages, les quartiers populaires) et dans la vie associative si riche du peuple. Et cela au profit et par le fait d'une manipulation et d'une approche technocratique des outils audiovisuels, qui seraient le fait de spécialistes détachés du peuple, qui concevraient programmes et émissions à partir de centres de décision lointains? (...) Le problème est de provoquer une réelle prise de conscience devant un tel phénomène, et tout d'abord chez les peuples d'Afrique eux-mêmes. Comment pourront-ils s'approprier l'audiovisuel, y exprimer la nouvelle culture qu'ils portent en attente? A quelles conditions les outils audiovisuels pourront-ils être détournés de leur usage actuel pour servir un projet libérateur de société? ${ }^{28}$

Trente ans après, force est de constater qu'il s'agissait là de vœux pieux. Le NOIIC (nouvel ordre international de l'information et de la communication) a été un total fiasco. Pour l'instant, la cyberculture est massivement anglo-saxonne et singulièrement nord-américaine, même si elle n'émane pas nécessairement des groupes multimédia. Très rapidement, on risque de voir un nouveau type de pauvreté, la «pauvreté d'information », élargissant plutôt que diminuant le fossé

${ }^{25}$ Op. cit., p. 132.

${ }^{26}$ «Faire confiance à la technique: Cyber-interview de Christian Huitema», Terminal, n 71/72 (Spécial Internet), 1997, p. 102.

${ }^{27}$ Michel Elie, «Internet et développement », Futuribles, n²14, 1996.

${ }^{28}$ Babakar Sine, «Audiovisuel et extraversion culturel», communication faite au colloque d'Epernay, 23-26 février 1977, p. 9 et 11. 
qui sépare les pays riches des pays pauvres ${ }^{29}$. Dernier exemple actuel, «la réussite d'Internet se fait aux dépens des hackers (développeurs informaticiens liés à la contre-culture américaine) qui en ont conçu les usages et, à terme, au profit des entreprises ${ }^{30}$. En ce qui concerne plus spécifiquement Internet, il s'inscrit malgré tout dans le projet de GII (Global Information Infrastructure), impulsé par les Etats-Unis, consistant à développer des «autoroutes de l'information» (un «réseau de réseaux »). Ce projet grandiose vise explicitement à la création d'un marché mondial plus généralisé, plus instantané, etc. «Il nous appartient, selon le vice président Al Gore, de construire une communauté mondiale dans laquelle les citoyens de pays voisins se regarderaient non comme des ennemis potentiels, mais comme des partenaires potentiels, tous membres d'une grande famille humaine reliés par une chaîne aux maillons de plus en plus nombreux (...) Elle rendra possible la création d'un marché mondial de l'information, où les consommateurs pourront acheter et vendre (...) La croissance mondiale peut s'enrichir de plusieurs centaines de milliards de dollars si nous nous engageons sur la voie de la GII $»^{31}$. Cela, grâce à la suppression en 1993 de la clause qui imposait un «usage acceptable» du réseau, c'est-à-dire qui en excluait en fait les activités commerciales $^{32}$. «Pour la nation, les bénéfices potentiels du projet sont immenses. La NII permettra aux entreprises américaines de relever victorieusement le défi de l'économie mondiale, ce qui entraînera la création d'emplois intéressants pour nos concitoyens et générera de la croissance pour l'ensemble de la nation (...) et maintenir l'avance technologique des Etats-Unis.» ${ }^{33}$ 《Faciliter les échanges électronique d'information pour développer la mondialisation du commerce et des affaires.» ${ }^{34}$ L'objectif américain se résume à: «plus de marchés pour nos produits et plus de produits pour le marché ». Il s'agit donc de réaliser un supermarché virtuel ou cybermarché, accomplissant l'impossible idéal du Marché ultra libéral: instantanéité, transparence, universalité. C'est l'hypermarché électronique à domicile par teleshopping ${ }^{35}$.

${ }^{29}$ Chiara Ottaviano, op. cit., p. 132.

so Yvonne Mignot-Lefebvre, op. cit., p. 35. Elle note encore: «Cette conjonction rare entre un mouvement social extrêmement fort et novateur et une nouvelle technologie de communication, la vidéo, était une efflorescence fragile à durée brève de vie. Ce phénomène s'est reproduit depuis avec moins d'ampleur pour d'autres techniques, plus cognitives comme le micro-ordinateur, et récemment pour ces objets nouveaux du désir que sont les multimédias, les mondes virtuels, le cyberespace... A chaque fois, se réduit le temps de mise au point des techniques tandis que se rétrécit l'espace ouvert aux développeurs et créatifs pour inventer de nouvelles pratiques », Ibid., p. 36.

${ }^{31}$ Discours du vice-président Al Gore à l'International Telecommunication Union, Buenos Aires, le 21 mars 1994. Extraits tirés de «Multimédia et communication à usage humain», Dossier pour un débat 56, Fondation pour le progrès de l'homme, 1996, p. 78-87. Pour l'anecdote, on peut noter que le père du vice-président $\mathrm{Al}$ Gore, inventeur du concept des autoroutes de la communication, dirigeait une société de construction d'autoroute... (voir Jean-loup Anthony, «Croissance d'entreprise ou développement humain », in CIEPAD, rencontres d'été 1995).

${ }^{32}$ Chiara Ottaviano, op. cit., p. 131.

${ }^{33}$ Multimédia, op. cit., p. 63.

${ }^{34}$ Ibid., p. 101, 6 millions d'Américains utilisent déjà le télétravail.

${ }^{35}$ Le rapport de 1'Union Européenne de Martin Bangemann qui calque celui d'Al Gore conclut: «Les pays qui entreront les premiers dans l'ère de l'information seront en mesure de dicter aux autres la suite des événements », Ibid., p. 113. 
L'interactivité d'internet a fait rêver de «démocratie sans frontière», de «parlement électronique» et de «télédémocratie» avec «nitizens» ou «citizenet ${ }^{36}$. Sans pousser jusqu'au délire de la cybersocialité, la question d'une solution technique aux apories de la démocratie directe peut sembler légitime. Toutefois, si certaines techniques peuvent résoudre certaines difficultés techniques de sa mise en œuvre, ces solutions ne peuvent sûrement pas se réaliser à l'intérieur du paradigme de la modernité marchande, qui a su déjà récupérer l'internet pour le supermarché électronique planétaire. Andrew Feenberg lui-même, d'ailleurs, en convient - dans une note, il est vrai. «De telles améliorations supposent des formes d'organisation plus participatives qui pourraient se révéler incompatibles avec le code technique capitaliste. ${ }^{37}$ C'est bien mon avis!

Chico Mendes fut assassiné le 22 décembre 1988 dans le fin fond de l'Amazonie, à Xapuri. Comme par hasard, le téléphone ne fonctionnait pas durant les heures qui s'ensuivirent et les portables n'existaient pas encore. Or il faut des heures de marche dans la forêt pour porter les nouvelles. Pourtant, la diffusion de l'information fut immédiate au Brésil et dans le monde entier. C'est que, si le web, à proprement parler, n'existait pas encore, internet, imaginé en 1964 par Paul Baran pour sauver les communications télématiques militaires en cas d'attaque soviétique, était utilisé dès les années 70 par les scientifiques pour échanger des informations; et les ONG Nord-américaines, très actives dans la région, fonctionnaient déjà en réseau interconnecté. De ce fait, la mobilisation nationale et internationale fut très rapide. Dans son édition du samedi 24 décembre, le «Jornal do Brasil » publiait sur une pleine page une interview du leader amazonien faite trois semaines auparavant.

Ainsi, grâce à une technique, inventée et mise au point par la CIA pour exercer un contrôle planétaire, le meurtre répugnant en forêt d'un résistant à l'oppression de l'économie-monde ne fut pas passé sous silence et, étant devenu un évènement global, a pu bouleverser la conscience planétaire. Depuis, le sous-commandant Marcos a fait mieux dans l'usage de la guérilla informatique pour populariser la révolte des Chiapas contre les «nouveaux maîtres du monde». Il est donc incontestable que certaines des techniques nouvelles donnent des instruments nouveaux au combat pour l'émancipation. Toutefois, les développements ultérieurs des exemples cités (la poursuite des expropriations en Amazonie, y compris sous le régime de Lula et la liquidation subreptice des leaders indiens des Chiapas) font douter de la possibilité de changer vraiment la donne grâce à la technique. D'ores et déjà, on voit internet devenir progressivement un instrument du «bit business » ou «cyberbusiness» avec l'invasion des «infomerciales» (scénarisations de la publicité).

Il y a une autre raison, plus profonde, qui vicie à la base le projet de télédémocratie globale et qui porte moins sur le volet technique que sur le volet «global». Je me méfie de tout projet universaliste, même radical ou subversif. J'ai tendance

${ }^{36}$ Jean-Louis Weissberg, «Internet, un récit utopique», Terminal, n 71/72 (Spécial Internet), 1997. Mark Dery, Vitesse virtuelle, Paris, Ed. Abbeville, 1997. Roland Jaccard, «Mythologies de la Cyberculture », Le monde des livres, 6.6.1997.

${ }^{37}$ Op. cit., p. 214. 
à y déceler des relents d'ethnocentrisme occidental ${ }^{38}$. «Pourquoi Dieu, se demande Raimon Panikkar, en détruisant le rêve de Babel, n'a-t-il pas voulu d'un gouvernement mondial, d'un marché mondial, d'une banque mondiale, d'une démocratie mondiale? Pourquoi a-t-il préféré, pour permettre aux hommes de communiquer, de petites huttes à échelle humaine, avec des fenêtres et des rues, et non des autoroutes de l'information? (...) Pour le philosophe, (la réponse) c'est pour que les rapports humains restent personnels $\gg^{39}$. L'idée qu'une humanité unifiée est la condition d'un fonctionnement harmonieux de la planète fait partie de la panoplie des fausses bonnes idées véhiculées par l'ethnocentrisme occidental ordinaire ${ }^{40}$. La diversité des cultures est sans doute la condition d'un commerce social paisible. En effet, chaque culture se caractérise par la spécificité de ses valeurs. Même s'il régnait un langage et une monnaie commune sur la planète, chaque culture leur accorderait des significations propres et partiellement différentes. On peut le vérifier sur le plan économique. Si les places de marché, les marchésrencontre ont été pendant des siècles sur presque tous les continents des lieux d'échange pacifique, de règlement des conflits, de circulation matrimoniale entre voisins et même entre ennemis, c'est que les transactions entre étrangers permises par l'intermédiation monétaire conservaient, en dépit de son anonymat relatif, les qualités du don réussi entre proches. Du fait des différences d'échelles de valeur, chacun en ressortait convaincu d'avoir fait une bonne affaire (voire d'avoir roulé son partenaire, lui-même persuadé d'avoir réussi le même coup!). Les marchés africains illustrent abondamment cette ruse du commerce pacifique entre cultures diverses. «En attribuant une valeur morale différente aux denrées échangées, écrit l'anthropologue Marco Aime, chacun des deux protagonistes s'en sortira comme le vainqueur suivant ses propres paramètres ${ }^{41}$. Ainsi, le malentendu interculturel est un «facilitateur» d'harmonie dans l'échange social en faisant règner la conviction partagée par chacun d'avoir obtenu son dû (voire un peu plus...). Il en va de même sur le plan politique. La démocratie, en particulier, ne peut probablement fonctionner que si la politie est de petite dimension et fortement ancrée dans ses valeurs propres. Dans une vision «pluriversaliste», les rapports entre les diverses polities au sein du village planétaire pourraient être réglés par une «démocratie des cultures ». Bien évidemment, il ne s'agit pas là d'un gouvernement mondial, mais d'une instance d'arbitrage minimale entre des polities souveraines de statuts très divers. Panikkar remarque encore:

Quand je m'oppose à un gouvernement mondial, je ne veux pas aller contre une harmonie universelle ou contre une forme de communication entre les hommes. Je reconnais que l'idée de gouvernement mondial est fantastique et je comprends que celui qui la

\footnotetext{
39 Le Monde du 2 avril 1996: «Qui a peur de perdre son identité l'a déjà perdue » (Entretien avec Henri Tincq).

${ }^{40}$ Ce que Denis Duclos qualifie fort justement de «délires d'universalité » à propos de «l'idéal d'une citoyenneté universelle et de son futur Etat planétaire, fantasme particulièrement présent chez les intellectuels en France », Société-monde, le temps des ruptures, Paris, La Découverte/M.A.U.S.S., 2002, p. 217.

${ }^{41}$ Marco Aime, La casa di nessuno, Torino, Bollati Boringhieri, 2002, p. 114. Voir aussi le dernier chapitre de notre livre « Justice sans limites», Paris, Fayard, 2003.
}

${ }^{38}$ Je me séparai déjà sur ce point de Castoriadis. La lecture de Takis Fotopoulos renforce mes doutes. 


\begin{abstract}
soutient ne veut pas être le président suprême de l'humanité, mais désire l'harmonie, la paix, la compréhension entre les peuples et voudrait peut-être supprimer comme moi l'Etat souverain. L'alternative que je cherche à offrir serait la biorégion, c'est-à-dire les régions naturelles où les troupeaux, les plantes, les animaux, les eaux et les hommes forment un ensemble unique et harmonieux. (...) Il faudrait arriver à un mythe qui permette la république universelle sans impliquer ni gouvernement, ni contrôle, ni police mondiale. Cela requiert un autre type de rapports entre les biorégions ${ }^{42}$.
\end{abstract}

Les technologies sophistiquées peuvent aider au fonctionnement technique d'une telle structure mais n'interfèrent pas sur son principe.

Finalement, on ne peut nier la puissance extraordinaire de l'instrument internet, en ce qu'elle change les données des luttes sociales. Le cas des versets sataniques de Salman Rushdie ou le scandale du corsage brodé au Coran de Claudia Schiffer dans une présentation de mode en Indonésie illustrent le changement d'échelle apporté par le couple mondialisation/médiatisation dont internet est une pièce maîtresse. Internet permet aux chercheurs du monde entier de fonctionner en réseau comme une seule communauté constituant une «intelligence collective», partageant des savoirs et échangeant en temps réel. Formidable! Mais comme le note Jean Loup Anthony: «Est-il vraiment utile qu'ils (les chercheurs) se mettent tous ensemble pour détruire plus rapidement la planète? Einstein se posait déjà cette question à la fin de sa vie $!^{43} \gg$. Toutefois, s'agissant de la lutte contre la mégamachine, on pourrait conclure assez lucidement avec le philosophe Jacques Poulain que nous tenons là la possibilité inouïe de faire partager de façon planétaire le constat de notre protestation impuissante.

Professeur émérite d'économie

Université de Paris XI

${ }^{42}$ «Politica e interculturalità », in Reinventare la politica, Città di Castello, L'Altrapagina, 1995, p. $22-23$.

${ }^{43}$ Jean-Loup Anthony, «Croissance d'entreprise ou développement humain», in CIEPAD, rencontres d'été 1995, p. 12. 